\title{
Cohomology of Frobenius Algebras and the Yang-Baxter Equation
}

\author{
J. Scott Carter* \\ University of South Alabama \\ Mohamed Elhamdadi \\ University of South Florida \\ Alissa S. Crans \\ Loyola Marymount University \\ Enver Karadayi \\ University of South Florida \\ Masahico Saito ${ }^{\dagger}$ \\ University of South Florida
}

November 21, 2018

Dedicated to the memory of Xiao-Song Lin

\begin{abstract}
A cohomology theory for multiplications and comultiplications of Frobenius algebras is developed in low dimensions in analogy with Hochschild cohomology of bialgebras based on deformation theory. Concrete computations are provided for key examples.

Skein theoretic constructions give rise to solutions to the Yang-Baxter equation using multiplications and comultiplications of Frobenius algebras, and 2-cocycles are used to obtain deformations of $R$-matrices thus obtained.
\end{abstract}

\section{Introduction}

Frobenius algebras are interesting to topologists as well as algebraists for numerous reasons including the following. First, 2-dimensional topological quantum field theories are formulated in terms of commutative Frobenius algebras (see [13]). Second, a Frobenius algebra structure exists on any finite-dimensional Hopf algebra with a left integral defined in the dual space. These Hopf algebras have found applications in topology through Kuperberg's invariant [14, 15], the Henning invariant [11, 17, and the theory of quantum groups from which the post-Jones invariants arise. Third, there is a 2-dimensional Frobenius algebra that underlies Khovanov's cohomology theory [12. See also [1].

Our interest herein is to extend the cohomology theories defined in [3, 4] to Frobenius algebras and thereby construct new solutions to the Yang-Baxter equation (YBE). We expect that there are connections among these cohomology theories that extend beyond their formal definitions. Furthermore, we anticipate topological, categorical, and/or physical applications because of the diagrammatic nature of the theory.

\footnotetext{
* Supported in part by NSF Grant DMS \#0603926.
}

${ }^{\dagger}$ Supported in part by NSF Grant DMS \#0603876. 
The 2-cocycle conditions of Hochschild cohomology of algebras and bialgebras can be interpreted via deformations of algebras [8]. In other words, a map satisfying the associativity condition can be deformed to obtain a new associative map in a larger vector space using 2-cocycles. The same interpretation can be applied to quandle cohomology theory [2, 5, 6]. A quandle is a set equipped with a self-distributive binary operation satisfying a few additional conditions that correspond to the properties that conjugation in a group enjoys. Quandles have been used in knot theory extensively (see [2] and references therein for more aspects of quandles). Quandles and related structures can be used to construct set-theoretic solutions (called $R$-matrices) to the Yang-Baxter equation (see, for example, [10] and its references). From this point of view, combined with the deformation 2-cocycle interpretation, a quandle 2-cocycle can be regarded as giving a cocycle deformation of an $R$-matrix. Thus we extend this idea to other algebraic constructions of $R$-matrices and construct new $R$-matrices from old via 2-cocycle deformations.

In [3, 4, new $R$-matrices were constructed via 2-cocycle deformations in two other algebraic contexts. Specifically, in [3], self-distributivity was revisited from the point of view of coalgebra categories, thereby unifying Lie algebras and quandles in these categories. Cohomology theories of Lie algebras and quandles were given via a single definition, and deformations of $R$-matrices were constructed. In [4, the adjoint map of Hopf algebras, which corresponds to the group conjugation map, was studied from the same viewpoint. A cohomology theory was constructed based on equalities satisfied by the adjoint map that are sufficient for it to satisfy the YBE.

In this paper, we present an analog for Frobenius algebras according to the following organization. After a brief review of necessary materials in Section 2, a cohomology theory for Frobenius algebras is constructed in Section 3 via deformation theory. Then Yang-Baxter solutions are constructed by skein methods in Section 4 , followed by deformations of $R$-matrices by 2-cocycles.

The reader should be aware that the composition of the maps is read in the standard way from right to left $(g f)(x)=g(f(x))$ in text and from bottom to top in the diagrams. In this way, when reading from left to right one can draw from top to bottom and when reading a diagram from top to bottom, one can display the maps from left to right. The argument of a function (or input object from a category) is found at the bottom of the diagram.

\section{Preliminaries}

A Frobenius algebra is an (associative) algebra (with multiplication $\mu: A \otimes A \rightarrow A$ and unit $\eta: k \rightarrow A$ ) over a field $k$ with a nondegenerate associative pairing $\beta: A \otimes A \rightarrow k$. Throughout this paper all algebras are finite-dimensional unless specifically stated otherwise. The pairing $\beta$ is also expressed by $\langle x \mid y\rangle=\beta(x \otimes y)$ for $x, y \in A$, and it is associative in the sense that $\langle x y \mid z\rangle=\langle x \mid y z\rangle$ for any $x, y, z \in A$.

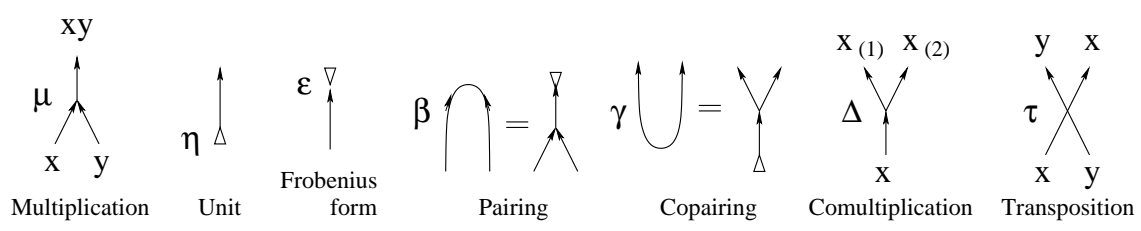

Figure 1: Diagrams for Frobenius algebra maps 
A Frobenius algebra $A$ has a linear functional $\epsilon: A \rightarrow k$, called the Frobenius form, such that the kernel contains no nontrivial left ideal. It is defined from $\beta$ by $\epsilon(x)=\beta(x \otimes 1)$, and conversely, a Frobenius form gives rise to a nondegenerate associative pairing $\beta$ by $\beta(x \otimes y)=\epsilon(x y)$, for $x, y \in A$.

A Frobenius form has a unique copairing $\gamma: k \rightarrow A \otimes A$ characterized by

$$
(\beta \otimes \mid)(\mid \otimes \gamma)=\mid=(\mid \otimes \beta)(\gamma \otimes \mid),
$$

where $\mid$ denotes the identity homomorphism on the algebra. We call this relation the cancelation of $\beta$ and $\gamma$. See the middle entry in the bottom row of Fig. 2, This notation will distinguish this function from the identity element $1=1_{A}=\eta\left(1_{k}\right)$ of the algebra that is the image of the identity of the ground field. A Frobenius algebra $A$ determines a coalgebra structure with $A$-linear (coassociative) comultiplication and the counit defined using the Frobenius form. The comultiplication $\Delta: A \rightarrow$ $A \otimes A$ is defined by

$$
\begin{aligned}
\Delta & =(\mu \otimes \mid)(\mid \otimes \gamma) \\
& =(\mid \otimes \mu)(\gamma \otimes \mid)
\end{aligned}
$$

The multiplication and comultiplication satisfy the following equality:

$$
\Delta \mu=(\mu \otimes \mid)(\mid \otimes \Delta)=(\mid \otimes \mu)(\Delta \otimes \mid)
$$

which we call the Frobenius compatibility condition.

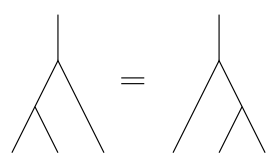

Associativity
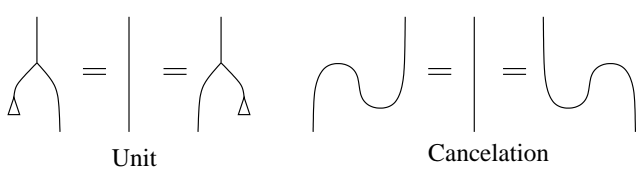

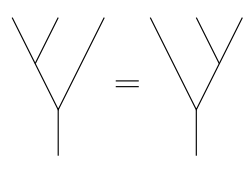

Coassociativity

Cancelation

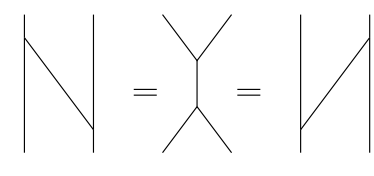

Compatibility

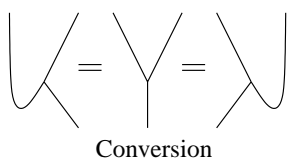

Figure 2: Equalities among Frobenius algebra maps

A Frobenius algebra is symmetric if the pairing is symmetric, meaning that $\beta(x \otimes y)=\beta(y \otimes x)$ for any $x, y \in A$. A Frobenius algebra is commutative if it is commutative as an algebra. It is known (13. Prop. 2.3.29) that a Frobenius algebra is commutative if and only if it is cocommutative as a coalgebra.

The map $\mu \Delta$ of a Frobenius algebra is called the handle operator, and corresponds to multiplication by a central element called the handle element $\delta_{h}=\mu \gamma(1)([13]$, page 128).

Any semisimple Hopf algebra gives rise to a Frobenius algebra structure (see, for example, 13], page 135). Let $H$ be a finite-dimensional Hopf algebra with multiplication $\mu$ and unit $\eta$. Then $H$ is semisimple if and only if the bilinear form $\beta_{a b}=\sum \mu_{c d}^{d} \mu_{a b}^{c}$ is nondegenerate. If $H$ is semisimple, then the above defined $\beta$ gives rise to a Frobenius pairing. In this case the Frobenius form (a counit of the Frobenius algebra structure) is defined by $\epsilon_{a}=\sum \mu_{c d}^{d}=T$, the trace of $H$. This counit and 
the induced comultiplication of the resulting Frobenius algebra structure should not be confused with the counit and the comultiplication of the original Hopf algebra. We thank Y. Sommerhausser for explaining these relationships to us.

We compute cohomology groups and the Yang-Baxter solutions for a variety of examples, and we review these mostly from [13]. From the point of view of TQFTs, the value $\epsilon \eta(1)$ corresponds to a sphere $S^{2}, \delta_{0}=\beta \gamma(1)$ to a torus $T^{2}$, and $\mu \Delta$ to adding an extra 1-handle to a tube, so we will compute these values and maps.

Example 2.1 Complex numbers with trigonometric comultiplication. Let $A=\mathbb{C}$ over $k=\mathbb{R}$ and let the basis be denoted by 1 and $i=\sqrt{-1}$. Then the Frobenius form $\epsilon$ defined by $\epsilon(1)=1$ and $\epsilon(i)=0$ gives rise to the comultiplication $\Delta$, which is Sweedler's trigonometric coalgebra with $\Delta(1)=1 \otimes 1-i \otimes i$, and $\Delta(i)=i \otimes 1+1 \otimes i$. We compute $\epsilon \eta(1)=1, \mu \Delta(1)=2 \eta(1)$, and $\mu \Delta(i)=2 i \eta(1)$ so that $\mu \Delta$ is multiplication by 2 , which is the handle element $\delta_{h}$. We also have $\delta_{0}=2$.

Example 2.2 Polynomial algebras. Polynomial rings $k[x] /\left(x^{n}\right)$ over a field $k$ where $n$ is a positive integer are Frobenius algebras. In particular, for $n=2$, the algebra $A=k[x] /\left(x^{2}\right)$ was used in the Khovanov homology of knots [12]. For $A=k[x] /\left(x^{2}\right)$, the Frobenius form $\epsilon: A \rightarrow k$ is defined by $\epsilon(x)=1$ and $\epsilon(1)=0$. This induces the comultiplication $\Delta: A \rightarrow A \otimes A$ determined by $\Delta(1)=1 \otimes x+x \otimes 1$ and $\Delta(x)=x \otimes x$. The handle element is $\delta_{h}=2 x$.

More generally for $A=k[x] /\left(x^{n}\right)$ and $\epsilon\left(x^{j}\right)=1$ for $j=n-1$ and 0 otherwise, the comultiplication is determined by $\Delta(1)=\sum_{i=0}^{n-1} x^{i} \otimes x^{n-1-i}$. We have $\mu \Delta(1)=n x^{n-1}$ and the handle element is $\delta_{h}=n x^{n-1}$.

Example 2.3 Group algebras. The group algebra $A=k G$ for a finite group $G$ over a field $k$ is a Frobenius algebra with $\epsilon(x)=0$ for any $G \ni x \neq 1$ and $\epsilon(1)=1$, where 1 is identified with the identity element. The induced comultiplication is given by $\Delta(x)=\sum_{y z=x} y \otimes z$.

One computes $\epsilon \eta(1)=1$ and $\mu \Delta(x)=|G| x$, where $|G|$ is the order of $G$. In particular, note that $\mu \Delta=\delta_{1} \mid$ (recall that $\mid$ denotes the identity map), where $\delta_{1}=|G|$ (the order of the group $G$ ), and $(\mu \Delta)^{n}=\delta_{1}^{n} \mid$ for any $n \in \mathbb{N}$, so that the handle element is $\delta_{h}=\delta_{1}=|G|$, and $\delta_{0}=|G|$.

There are other Frobenius forms on the group algebra A (again from [13]). For example, for $A=k G$ where $G$ is the symmetric group on three letters, $A=k\langle x, y\rangle /\left(x^{2}-1, y^{2}-1, x y x=y x y\right)$, and $\epsilon(x y x)=1$ and otherwise zero, is a Frobenius form and the handle element is $2(x y x+x+y)$.

Example $2.4 q$-Commutative polynomials. Let $X=k\langle x, y\rangle /\left(x^{2}, y^{2}, y x-q x y\right)$ where $q=$ $-A^{-2}$ for $A \in k$ with polynomial multiplication and $\epsilon(x y)=i A$, and zero for other basis elements. Then

$$
\gamma(1)(=\Delta \eta(1))=i A(x \otimes y)-i A^{-1}(y \otimes x)-i A^{-1}(x y \otimes 1+1 \otimes x y) .
$$

One computes

$$
\begin{aligned}
\Delta(x) & =-i A^{-1}(x \otimes x y+x y \otimes x), \\
\Delta(y) & =-i A^{-1}(y \otimes x y+x y \otimes y), \\
\Delta(x y) & =-i A^{-1}(x y \otimes x y) .
\end{aligned}
$$

The handle element is $\delta_{h}=i A^{-1}\left(A-A^{-1}\right)^{2}(x y)$. 


\section{Deformations and cohomology groups}

We describe the deformation theory of multiplication and comultiplication for Frobenius algebras mimicking [8], [16], and our approach in [3, 4]. This approach will yield the definition of 2-cocycles. We will define the chain complex for Frobenius algebras with chain groups in low dimensions [8, 16]. We expect topological applications in low dimensions. The differentials are defined via diagrammatically defined identities among relations.

\subsection{Deformations}

In [16], deformations of bialgebras were described. We follow that formalism and give deformations of multiplications and comultiplications of Frobenius algebras. A deformation of $A=(V, \mu, \Delta)$ is a $k[[t]]$-Frobenius algebra $A_{t}=\left(V_{t}, \mu_{t}, \Delta_{t}\right)$, where $V_{t}=V \otimes k[[t]]$ and $V_{t} /\left(t V_{t}\right) \cong V$. Deformations of $\mu$ and $\Delta$ are given by $\mu_{t}=\mu+t \mu_{1}+\cdots+t^{n} \mu_{n}+\cdots: V_{t} \otimes V_{t} \rightarrow V_{t}$ and $\Delta_{t}=\Delta+t \Delta_{1}+\cdots+t^{n} \Delta_{n}+\cdots$ : $V_{t} \rightarrow V_{t} \otimes V_{t}$ where $\mu_{i}: V \otimes V \rightarrow V, \Delta_{i}: V \rightarrow V \otimes V, i=1,2, \cdots$, are sequences of maps. Suppose $\bar{\mu}=\mu+\cdots+t^{n} \mu_{n}$ and $\bar{\Delta}=\Delta+\cdots+t^{n} \Delta_{n}$ satisfy the Frobenius conditions (associativity, compatibility, and coassociativity) $\bmod t^{n+1}$, and suppose that there exist $\mu_{n+1}: V \otimes V \rightarrow V$ and $\Delta_{n+1}: V \rightarrow V \otimes V$ such that $\bar{\mu}+t^{n+1} \mu_{n+1}$ and $\bar{\Delta}+t^{n+1} \Delta_{n+1}$ satisfy the Frobenius algebra conditions $\bmod t^{n+2}$. Define $\xi_{1} \in \operatorname{Hom}\left(V^{\otimes 3}, V\right), \xi_{2}, \xi_{2}^{\prime} \in \operatorname{Hom}\left(V^{\otimes 2}, V^{\otimes 2}\right)$, and $\xi_{3} \in \operatorname{Hom}\left(V, V^{\otimes 3}\right)$ by:

$$
\begin{array}{rll}
\bar{\mu}(\bar{\mu} \otimes \mid)-\bar{\mu}(\mid \otimes \bar{\mu}) & =t^{n+1} \xi_{1} & \bmod t^{n+2}, \\
\bar{\Delta} \bar{\mu}-(\bar{\mu} \otimes \mid)(\mid \otimes \bar{\Delta}) & =t^{n+1} \xi_{2} & \bmod t^{n+2}, \\
\bar{\Delta} \bar{\mu}-(\mid \otimes \bar{\mu})(\bar{\Delta} \otimes \mid) & =t^{n+1} \xi_{2}^{\prime} & \bmod t^{n+2}, \\
(\bar{\Delta} \otimes \mid) \bar{\Delta}-(\mid \otimes \bar{\Delta}) \bar{\Delta} & =t^{n+1} \xi_{3} & \bmod t^{n+2}
\end{array}
$$

Remark 3.1 The operators in the quadruple $\left(\xi_{1}, \xi_{2}, \xi_{2}^{\prime}, \xi_{3}\right)$ form the primary obstructions to formal deformations of multiplication and comultiplication of a Frobenius algebra [16].

For the associativity of $\bar{\mu}+t^{n+1} \mu_{n+1} \bmod t^{n+2}$ we obtain:

$$
\left(\bar{\mu}+t^{n+1} \mu_{n+1}\right)\left(\left(\bar{\mu}+t^{n+1} \mu_{n+1}\right) \otimes \mid\right)-\left(\bar{\mu}+t^{n+1} \mu_{n+1}\right)\left(\mid \otimes\left(\bar{\mu}+t^{n+1} \mu_{n+1}\right)\right)=0 \bmod t^{n+2}
$$

which is equivalent by degree calculations to:

$$
\left(d^{2,1}\left(\mu_{n+1}\right)=\right) \quad \mu\left(\mid \otimes \mu_{n+1}\right)+\mu_{n+1}(\mid \otimes \mu)-\mu\left(\mu_{n+1} \otimes \mid\right)-\mu_{n+1}(\mu \otimes \mid)=\xi_{1},
$$

where $d^{2,1}$ is one of the differentials we will define in the following section. Similarly, from the Frobenius compatibility condition and coassociativity we obtain

$$
\begin{aligned}
\left(d_{(1)}^{2,2}\left(\mu_{n+1}, \Delta_{n+1}\right)=\right) & \Delta \mu_{n+1}+\Delta_{n+1} \mu-(\mu \otimes \mid)\left(\mid \otimes \Delta_{n+1}\right)-\left(\mu_{n+1} \otimes \mid\right)(\mid \otimes \Delta)=\xi_{2}, \\
\left(d_{(2)}^{2,2}\left(\mu_{n+1}, \Delta_{n+1}\right)=\right) & \Delta \mu_{n+1}+\Delta_{n+1} \mu-(\mid \otimes \mu)\left(\Delta_{n+1} \otimes \mid\right)-\left(\mid \otimes \mu_{n+1}\right)(\Delta \otimes \mid)=\xi_{2}^{\prime}, \\
\left(d^{2,3}\left(\Delta_{n+1}\right)=\right) & (\Delta \otimes \mid) \Delta_{n+1}+\left(\Delta_{n+1} \otimes \mid\right) \Delta-(\mid \otimes \Delta) \Delta_{n+1}-\left(\mid \otimes \Delta_{n+1}\right) \Delta=\xi_{3},
\end{aligned}
$$

where there are two types of compatibility conditions for $d^{2,2}$.

In summary we proved the following: 
Lemma 3.2 The maps $\bar{\mu}+t^{n+1} \mu_{n+1}$ and $\bar{\Delta}+t^{n+1} \Delta_{n+1}$ satisfy the associativity, coassociativity and Frobenius compatibility conditions mod $t^{n+2}$ if and only if the equalities (1), (2), (3) and (4) are satisfied.

\subsection{Chain groups}

Let $A$ be a Frobenius algebra. We define chain groups as follows.

$$
\begin{aligned}
C_{f}^{n, i}(A ; A) & =\operatorname{Hom}\left(A^{\otimes(n+1-i)}, A^{\otimes i}\right), \\
C_{f}^{n}(A ; A) & =\oplus_{0<i \leq n} C_{f}^{n, i}(A ; A) .
\end{aligned}
$$

Specifically, chain groups in low dimensions of our concern are:

$$
\begin{aligned}
& C_{f}^{1}(A ; A)=\operatorname{Hom}(A, A), \\
& C_{f}^{2}(A ; A)=\operatorname{Hom}\left(A^{\otimes 2}, A\right) \oplus \operatorname{Hom}\left(A, A^{\otimes 2}\right), \\
& C_{f}^{3}(A ; A)=\operatorname{Hom}\left(A^{\otimes 3}, A\right) \oplus \operatorname{Hom}\left(A^{\otimes 2}, A^{\otimes 2}\right) \oplus \operatorname{Hom}\left(A, A^{\otimes 3}\right) .
\end{aligned}
$$

In the remaining sections we will define differentials that are homomorphisms between the chain groups:

$$
d_{f}^{n, i}=d^{n, i}: C_{f}^{n}(A ; A) \rightarrow C_{f}^{n+1, i}(A ; A)\left(=\operatorname{Hom}\left(A^{\otimes(n+2-i)}, A^{\otimes i}\right)\right)
$$

which will be defined individually for $n=1,2,3$ and for $i$ with $0 \leq i \leq n$, and

$$
\begin{aligned}
D_{1} & =d^{1,1}-d^{1,2}: C_{f}^{1}(A ; A) \rightarrow C_{f}^{2}(A ; A), \\
D_{2}^{(i)} & =d^{2,1}+d_{(i)}^{2,2}+d^{2,3}: C_{f}^{2}(A ; A) \rightarrow C_{f}^{3}(A ; A), \\
D_{3} & =d^{3,1}+d^{3,2}+d^{3,3}+d^{3,4}: C_{f}^{3}(A ; A) \rightarrow C_{f}^{4}(A ; A) .
\end{aligned}
$$

Define $C_{f}^{0}(A ; A)=0$ by convention. From now on the subscripts $f$ for differentials are omitted for simplicity if no confusion arises.

\subsection{First differentials}

By analogy with the differential for associative multiplication, we make the following definition:

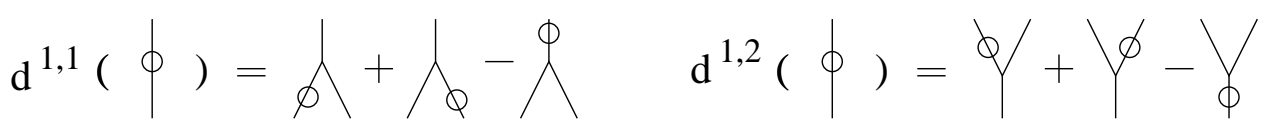

Figure 3: First differentials

Definition 3.3 The first differentials $d^{1,1}: C_{f}^{1,1}(A ; A) \rightarrow C_{f}^{2,1}(A ; A)$ and $d^{1,2}: C_{f}^{1,1}(A ; A) \rightarrow$ $C_{f}^{2,2}(A ; A)$ are defined, respectively, by

$$
\begin{aligned}
& d^{1,1}(h)=\mu(h \otimes \mid)+\mu(\mid \otimes h)-h \mu, \\
& d^{1,2}(h)=(h \otimes \mid) \Delta+(\mid \otimes h) \Delta-\Delta h .
\end{aligned}
$$

Then define $D_{1}: C_{f}^{1}(A ; A) \rightarrow C_{f}^{2}(A ; A)$ by $D_{1}=d^{1,1}-d^{1,2}$. 
Diagrammatically, we represent $d^{1, i}$ for $i=1,2$, as depicted in Fig. 3. A map $h \in C_{f}^{1,1}(A ; A)$ is represented by a white circle on a vertical string and the multiplication and comultiplication are depicted by two distinct trivalent vertices as before.

\subsection{Second Differentials}

An analogy with deformation theory when $\bar{\mu}=\mu+t \phi_{1}$ and $\bar{\Delta}=\Delta+t \phi_{2}$ gives the following.

Definition 3.4 Define the second differentials by:

$$
\begin{aligned}
d^{2,1}\left(\phi_{1}, \phi_{2}\right)=d^{2,1}\left(\phi_{1}\right) & =\mu\left(\phi_{1} \otimes \mid\right)+\phi_{1}(\mu \otimes \mid)-\mu\left(\mid \otimes \phi_{1}\right)-\phi_{1}(\mid \otimes \mu), \\
d_{(1)}^{2,2}\left(\phi_{1}, \phi_{2}\right) & =\Delta \phi_{1}+\phi_{2} \mu-\left(\phi_{1} \otimes \mid\right)(\mid \otimes \Delta)-(\mu \otimes \mid)\left(\mid \otimes \phi_{2}\right), \\
d_{(2)}^{2,2}\left(\phi_{1}, \phi_{2}\right) & =\Delta \phi_{1}+\phi_{2} \mu-\left(\mid \otimes \phi_{1}\right)(\Delta \otimes \mid)-(\mid \otimes \mu)\left(\phi_{2} \otimes \mid\right), \\
d^{2,3}\left(\phi_{1}, \phi_{2}\right)=d^{2,3}\left(\phi_{2}\right) & =\left(\phi_{2} \otimes \mid\right) \Delta+(\Delta \otimes \mid) \phi_{2}-\left(\mid \otimes \phi_{2}\right) \Delta-(\mid \otimes \Delta) \phi_{2} .
\end{aligned}
$$

Diagrams for 2-cochain and 2-differentials are depicted in Fig. 4 for $d^{2,1}$ and Fig. 5 for $d_{(1)}^{2,2}$ and $d_{(2)}^{2,2}$, respectively, where $\phi_{1} \in C_{f}^{2,1}(A ; A)$ and $\phi_{2} \in C_{f}^{2,2}(A ; A)$ are represented by black triangles on two different trivalent vertices. The diagrams for $d^{2,3}$ are upside-down pictures of Fig. 4 .

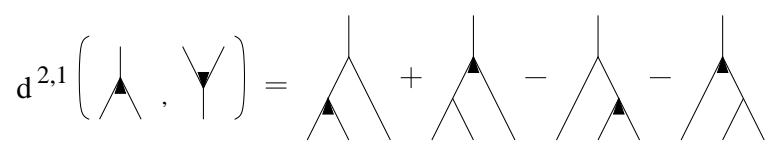

Figure 4: A 2-differential $d^{2,1}$

Then define $D_{2}: C_{f}^{2}(A ; A) \rightarrow C_{f}^{2}(A ; A)$ by $D_{2}=D_{2}^{(i)}=d^{2,1}+d_{(i)}^{2,2}+d^{2,3}$ for either $i=1$ or 2. Recall that for higher dimensions, differentials depend on this choice of $i=1$ or 2 at this exact dimension 2 for $d_{(1)}^{2,2}$ or $d_{(2)}^{2,2}$, due to compatibility. To avoid duplication in exposition, we choose, once and for all, $i=1$. The case for $i=2$ will be clear, as all the maps corresponding to $(\mu \otimes \mid)(\mid \otimes \Delta)$ in the case $i=1$ are simply replaced by those corresponding to $(\mid \otimes \mu)(\Delta \otimes \mid)$ in the case $i=2$. Diagrammatically they are mirror images. In the case $i=1$, the map looks like the letter "N" and its mirror in the case $i=2$.

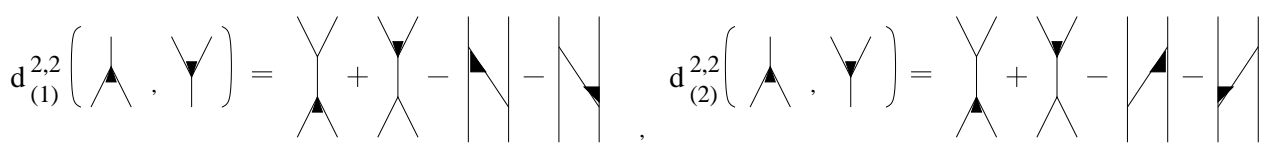

Figure 5: 2-differentials $d_{(1)}^{2,2}$ and $d_{(2)}^{2,2}$

Theorem $3.5 D_{2} D_{1}=0$.

Proof. This follows from direct calculations and also can be seen from diagrams as depicted in Fig. 6.

From the definition of the primary obstruction in Remark 3.1, the definition of the second differential implies the following. 


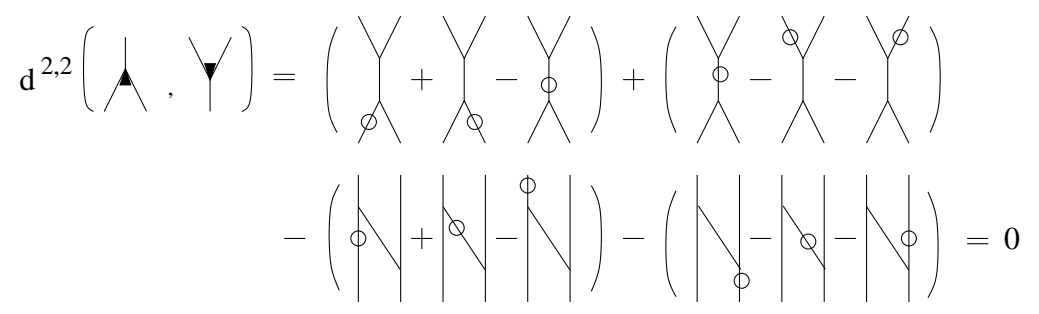

Figure 6: The 2-cocycle condition for a 2-coboundary: $d_{(1)}^{2,2}\left(d^{1,1}, d^{1,2}\right)=0$

Lemma 3.6 For a deformation $\left(\bar{\mu}+\mu_{n+1}, \bar{\Delta}+\Delta_{n+1}\right)$ of the multiplication and comultiplication of a Frobenius algebra $A$ defined in Section 3.1 and the primary obstruction $\left(\xi_{1}, \xi_{2}, \xi_{2}^{\prime}, \xi_{3}\right)$ defined in Remark [3.1, the relations $D_{2}^{(1)}\left(\mu_{n+1}, \Delta_{n+1}\right)=\left(\xi_{1}, \xi_{2}, \xi_{3}\right)$ and $D_{2}^{(2)}\left(\mu_{n+1}, \Delta_{n+1}\right)=\left(\xi_{1}, \xi_{2}^{\prime}, \xi_{3}\right)$ hold.

In particular, the primary obstructions vanish if and only if $\left(\mu_{n+1}, \Delta_{n+1}\right)$ is a 2-cocycle.

\subsection{Third differentials}

For simplicity we continue to use the case $d_{(1)}^{2,2}$ corresponding to $(\mu \otimes \mid)(\mid \otimes \Delta)$ for higher dimensions, and omit subscripts (1) from all the differentials.

Definition 3.7 Define the third differentials by:

$$
\begin{aligned}
& d^{3,1}\left(\xi_{1}, \xi_{2}, \xi_{3}\right)=\mu\left(\xi_{1} \otimes \mid\right)+\xi_{1}(|\otimes \mu \otimes|)+\mu\left(\mid \otimes \xi_{1}\right)-\xi_{1}(\mu \otimes \mid)-\xi_{1}(\mid \otimes \mu), \\
& d^{3,2}\left(\xi_{1}, \xi_{2}, \xi_{3}\right)=\Delta \xi_{1}+\xi_{2}(\mid \otimes \mu)+(\mu \otimes \mid)\left(\mid \otimes \xi_{2}\right)-\xi_{2}(\mu \otimes \mid)-\left(\xi_{1} \otimes \mid\right)(\mid \otimes 2 \otimes \Delta), \\
& d^{3,3}\left(\xi_{1}, \xi_{2}, \xi_{3}\right)=\xi_{3} \mu+(\mid \otimes \Delta) \xi_{2}+\left(\mid \otimes \xi_{2}\right)(\Delta \otimes \mid)-(\Delta \otimes \mid) \xi_{2}-(\mid \otimes 2 \otimes \mu)\left(\xi_{3} \otimes \mid\right), \\
& d^{3,4}\left(\xi_{1}, \xi_{2}, \xi_{3}\right)=\left(\xi_{3} \otimes \mid\right) \Delta+(|\otimes \Delta \otimes|) \xi_{3}+\left(\mid \otimes \xi_{3}\right) \Delta-\xi_{1}(\mu \otimes \mid)-(\mid \otimes \Delta) \xi_{3} .
\end{aligned}
$$

and recall that

$$
D_{3}=d^{3,1}+d^{3,2}+d^{3,3}+d^{3,4}: C_{f}^{3}(A ; A) \rightarrow C_{f}^{4}(A ; A) .
$$

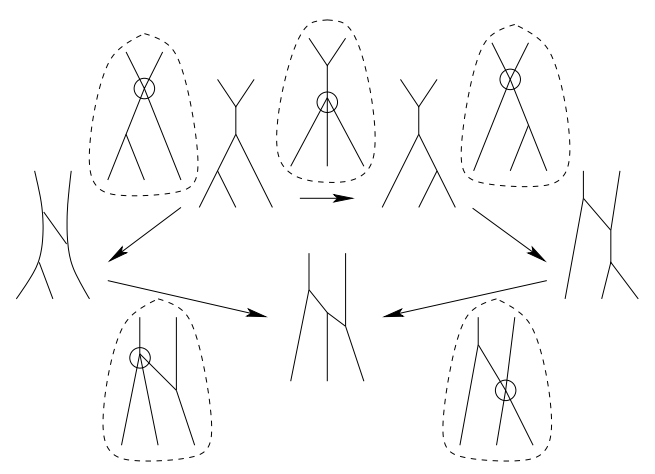

Figure 7: Deriving a 3-differential $d^{3,2}$

The differentials $d^{3,1}$ and $d^{3,4}$ are the Hochschild differentials that are derived from the pentagon conditions for associativity and coassociativity, respectively. The differentials $d^{3,2}$ and $d^{3,3}$ are dual 


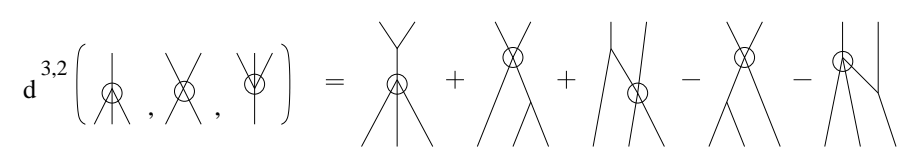

Figure 8: A 3-differential $d^{3,2}$

in the sense that once one is described via graphs, then the other is a mirror reflection in a horizontal line. We turn to describe the third differential $d^{3,2}$. Recall that the 2-cocycle conditions for a pair of maps $\left(\phi_{1}, \phi_{2}\right)$ are obtained by replacing incidences of multiplication and comultiplication by $\phi_{1}$ and $\phi_{2}$ in the associativity, compatibility, and coassociativity conditions. The equalities are depicted as equalities of graphs that contain trivalent vertices. The equalities themselves, then, can be depicted as 4 -valent vertices. There are three such vertices: a $(3,1)$ vertex corresponds to associativity $(3$ up, 1 down), a $(2,2)$ vertex corresponds to either one of the compatibility conditions, and a $(1,3)$ vertex corresponds to coassociativity. At the top left of Fig. 7 a graph is depicted that represents the composition $(\Delta)(\mu)(\mu \otimes \mid)$. There are two ways of deforming this graph using associativity or the $N$-compatibility condition. These transformations are depicted in the figure as an encircled $(3,1)$ vertex on the right arrow or an encircled $(2,2)$ vertex on the left arrow. Such vertices represent $\xi_{1}$ or $\xi_{2}$, respectively. Continuing around the diagram by choosing a new point at which an identity could be applied, we obtain a pentagon condition which can be derived from the pentagon condition on parentheses structures that gives the Biedenharn-Elliot identity (see [7], for example). Now read clockwise in a cycle around the diagram to write down the 3-coboundary of the pair $\left(\xi_{1}, \xi_{2}\right)$; motion against the direction of the arrows results in negative coefficients.

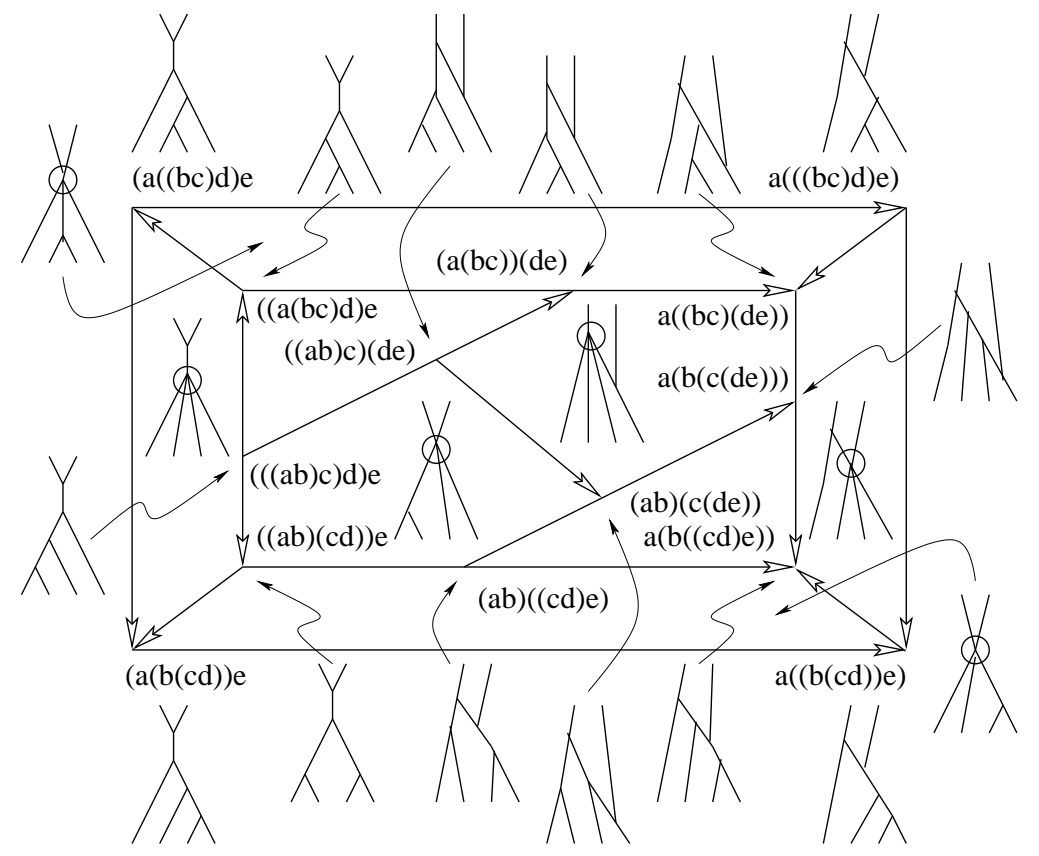

Figure 9: Diagrams for $d^{4,2}$ 
The pentagon itself can be labeled by a $(3,2)$-vertex that represents a 4 -cochain $\zeta_{2} \in C_{f}^{3,2}(A ; A)$. The corresponding 4-coboundary is a linear combination of the cells in the Stasheff polyhedron depicted in Fig. 9 in which the cell corresponding to $\zeta_{2}$ appears as the bottom pentagon, which is drawn as a trapezoid in the figure.

Theorem $3.8 D_{3} D_{2}=0$.

Proof. Since $d^{3,1}$ and $d^{3,4}$ are the same as the Hochschild differentials of bialgebras, and $d^{3,2}$ and $d^{3,3}$ are dual to each other, we only consider the case of $d^{3,2}\left[D_{2}\left(\phi_{1}, \phi_{2}\right)\right]=0$. This again follows from direct calculations, and can also be seen from diagrams as in Fig. 10, In the figure, each term of $d^{3,2}$ is replaced by the terms of $D_{2}\left(\phi_{1}, \phi_{2}\right)$, and the canceling terms are indicated by matching integers at the top-left corners of the diagrams.

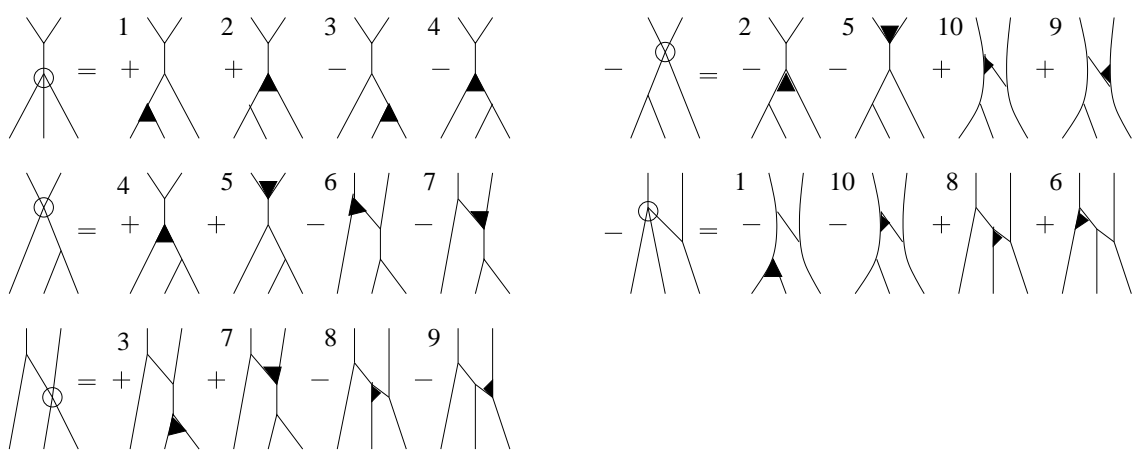

Figure 10: $D_{3} D_{2}=0$

From point of view of deformation theory, continuing from Lemma 3.6, we obtain the following.

Lemma 3.9 The primary obstruction $\left(\xi_{1}, \xi_{2}, \xi_{3}\right)$ and $\left(\xi_{1}, \xi_{2}^{\prime}, \xi_{3}\right)$ to a deformation of the multiplication and comultiplication of a Frobenius algebra $A$ defined in Remark 3.1 give a 3-cocycle: $D_{3}\left(\xi_{1}, \xi_{2}, \xi_{3}\right)=0$, and $D_{3}\left(\xi_{1}, \xi_{2}^{\prime}, \xi_{3}\right)=0$.

\subsection{Fourth differentials}

Recall that two third differentials, $d^{3,1}$ and $d^{3,4}$, are the same as the Hochschild differentials of bialgebras, and $d^{3,2}$ and $d^{3,3}$ are dual to each other. In fact, even the latter two have the same diagrammatic aspect of being a pentagon. Indeed, the graphs involved have two edges pointing upward instead of a single edge. Since the same phenomena continues to the fourth differentials, we only describe the formula for $d^{4,2}$. As before, $d^{4,1}$ and $d^{4,5}$ are Hochschild differentials, and $d^{4,4}$ is dual to $d^{4,2}$, and $d^{4,3}$ is a self-dual symmetric version of $d^{4,2}$. Thus an explicit formula, together with diagrammatic explanations for $d^{4,2}$, would suffice.

Definition 3.10 Define $d^{4,2}$ as follows:

$$
\begin{aligned}
d^{4,2}\left(\zeta_{1}, \zeta_{2}, \zeta_{3}, \zeta_{4}\right)= & \Delta \zeta_{1}+\zeta_{2}(|\otimes| \otimes \mu)+(\mu \otimes \mid)\left(\mid \otimes \zeta_{2}\right) \\
& -\left(\zeta_{1} \otimes \mid\right)(|\otimes| \otimes \mid \otimes \Delta)-\zeta_{2}(\mu \otimes|\otimes|)-\zeta_{2}(|\otimes \mu \otimes|) .
\end{aligned}
$$


Figure 9 depicts the Stasheff polytope (or associahedron) with vertices, edges, and faces having labels adapted to the current purposes: to formulate the fourth differential. The polytope consists of six pentagons and three squares. In the current drawing, four of the pentagons are drawn as trapezoids with their fifth vertex appearing as an interior point on each of the shorter of the parallel edges. The boundary of the planar figure represents one of the three squares in the polytope. The drawing, then, is a distortion of a central projection through this face. The other two squares are in the northwestern and southeastern center of the figure while the other two pentagons are in the southwest/northeast corners of the center. To each vertex, we associate a parenthesization of the five letters $a, b, c, d, e$ so that the resulting string represents a composition of binary products. Each edge corresponds to a single change of parenthesis such as $(a b) c$ to $a(b c)$. Meanwhile, a parenthesis structure can also be represented by a tree diagram. For example, $(a b) c$ and $a(b c)$ are represented by the first and third terms drawn in the RHS in Fig. 4, respectively, where for the moment you should ignore the black triangles. The pentagons in Fig. 9 are formed as a cycle of such regroupings. The edges, vertices, and faces here are indicated by tree diagrams which have two upward pointing branches and four downward pointing roots. To form correspondences between these tree and the groupings of $a, b, c, d, e$ bend the right branches down.

The pentagon in Fig. 7 represents a 4 -cochain $\zeta_{2} \in C^{4,2}(A ; A)$, which is also represented by a 5 -valent circled vertex with three edges pointing down and two up. When a 4-cochain $\zeta_{2}$ is identified with a pentagon, it is regarded as a homotopy of a path that starts from the three edges that are three consecutive arrows of the same direction, and sweeps the pentagonal face to the two remaining edge arrows. In Fig. 7, the three starting edges and two terminal edges both start at the top left corner of the pentagon and end at the bottom center. If a homotopy starts from two arrows instead, and sweeps the pentagon and ends at the three arrows, then it is regarded as the negative of the corresponding cochain, $-\zeta_{2}$.

In Fig. 9, there is a unique vertex $v_{0}$ from which all three arrows point out, which is assigned the parenthesized term $(((a b) c) d) e$. The unique vertex $v_{1}$ into which three arrows point is assigned $a(b((c d) e))$. There are edge paths that follow the directions of arrows that go from $v_{0}$ to $v_{1}$. Such an edge path $\gamma$ is homotopic to itself through pentagonal and square faces (including the "outside square" in Fig. 9) of the Stasheff polytope sweeping the entire sphere of the polytope. By formulating each pentagonal face in terms of the maps represented by tree diagrams, we obtain the formula for $d^{4,2}$.

Theorem $3.11 D_{4} D_{3}=0$.

Sketch of proof. This again follows from direct calculations and can also be seen from diagrams in Fig. 9. Here we explain how we see cancelations on diagrams. In computing $D_{4} D_{3}$, the terms in expressions in $D_{4}$ have their $\zeta$-factors systematically replaced by expressions that appear in the edges of the boundary of each pentagon, but each edge is labeled by an operator involving an $\xi$ as indicated, for example, in Fig. 7. Since each edge is the boundary of exactly two regions in the associahedron, the terms cancel. Note that if one of the bounded cells is a square, then there is a corresponding identity among those four terms - commutativity of distant tensor operators that causes the boundaries to cancel. The remaining details are left to the reader.

Remark 3.12 While we have not followed through all the details of such a construction, it seems reasonable to parametrize all the higher differentials in terms of the cells of the higher-dimensional 
Stasheff polytopes. The coboundaries are parametrized by the boundaries of these cells, and that the square of the differential is trivial will follow from the codimension 1 boundaries appearing on exactly two faces with opposite orientations. In this way, the cohomology of Frobenius algebras should be defined in all dimensions.

\subsection{Cohomology Groups}

For convenience define $C_{f}^{0}(A ; A)=0$ and $D_{0}=0: C_{f}^{0}(A ; A) \rightarrow C_{f}^{1}(A ; A)$. Then Theorems 3.5 and 3.8 are summarized as:

Theorem $3.13 \mathcal{C}=\left(C^{n}, D_{n}\right)_{n=0,1,2,3,4}$ is a chain complex.

This enables us to define:

Definition 3.14 The Frobenius n-coboundary, cocycle, and cohomology groups are defined by:

$$
\begin{aligned}
B_{f}^{n}(A ; A) & =\operatorname{Image}\left(D_{n-1}\right) \\
Z_{f}^{n}(A ; A) & =\operatorname{Ker}\left(D_{n}\right) \\
H_{f}^{n}(A ; A) & =Z_{f}^{n}(A ; A) / B_{f}^{n}(A ; A)
\end{aligned}
$$

for $n=1,2,3,4$.

The lemma below follows from the definitions.

Lemma 3.15 Let $A$ be a Frobenius algebra with $1=1_{A}=\eta 1_{k}$. Then:

(i) $d^{1,1}(h)(1 \otimes 1)=h(1)$, and

(ii) $d^{1,1}(h)(1 \otimes x)=d^{1,1}(h)(x \otimes 1)=h(1) x$, for any $x \in A$.

(iii) If $\gamma(1)=1 \otimes x+x \otimes 1$ for some $x \in A$, and $h \in Z_{f}^{1}(A ; A)$, then $h(x)=\alpha \cdot 1$, for some constant $\alpha$ such that $2 \alpha=0 \in k$. In particular, $h(x)=0$ if $\operatorname{char}(k) \neq 2$.

Proof. One computes $d^{1,1}(h)(1 \otimes 1)=h(1)$, and (ii) follows from direct calculations. For (iii), using $d^{1,2}(h)(1)=0$, we obtain $h(x) \otimes 1+1 \otimes h(x)=0$, which implies the statement.

Lemma 3.16 If a Frobenius algebra $A$ is commutative and $d^{2,1}\left(\phi_{1}\right)=0$, then for any $x \in A$, the following hold:

$$
\begin{aligned}
& x \phi_{1}(1 \otimes 1)=\phi_{1}(1 \otimes x)=\phi_{1}(x \otimes 1) \\
& \phi_{1}\left(x^{2} \otimes x\right)=\phi_{1}\left(x \otimes x^{2}\right), \quad \phi_{1}\left(1 \otimes x^{2}\right)=x \phi_{1}(1 \otimes x) .
\end{aligned}
$$

Proof. One computes $d^{2,1}\left(\phi_{1}\right)(a \otimes b \otimes c)$ for $(a, b, c)=(1,1, x)$ and $(x, 1,1)$, for the first set of equations, and $(x, x, x),(1, x, x)$ for the second set, respectively. The other choices using two elements $\{1, x\}$ do not give additional conditions. 


\subsection{Examples}

In this subsection we choose $d_{(1)}^{2,2}$ for the chain complex to compute. Throughout this section, the symbols $\gamma_{c}^{a b}$ and $\lambda_{b c}^{a}$ indicate the structure constants of comultiplication and multiplication for the algebras in question.

Example 3.17 For the example of complex numbers in Example 2.1, we have

$$
H_{f}^{1}(\mathbb{C} ; \mathbb{C})=0, \quad Z_{f}^{2}(\mathbb{C} ; \mathbb{C})=\mathbb{R}^{6}, \quad H_{f}^{2}(\mathbb{C} ; \mathbb{C})=\mathbb{R}^{4}
$$

Proof. By Lemma 3.15 (i), for $h \in Z_{f}^{1}(\mathbb{C} ; \mathbb{C})$, we have $h(1)=0$, and from $d^{1,1}(h)(i \otimes i)=0$, we obtain $h(i)=0$. Thus we obtain $Z_{f}^{1}(\mathbb{C} ; \mathbb{C})=0=H_{f}^{1}(\mathbb{C} ; \mathbb{C})$. This also implies that $B_{f}^{2}(\mathbb{C} ; \mathbb{C}) \cong \mathbb{R}^{2}$.

By Lemma 3.16, we have

$$
i \phi_{1}(1 \otimes 1)=\phi_{1}(1 \otimes i)=\phi_{1}(i \otimes 1)
$$

from $d^{2,1}\left(\phi_{1}\right)=0$. Hence we write

$$
\phi_{1}(1 \otimes i)=\phi_{1}(i \otimes 1)=\lambda 1+\lambda^{\prime} i
$$

for some $\lambda, \lambda^{\prime} \in \mathbb{R}$, and then $\phi_{1}(1 \otimes 1)=\lambda^{\prime} 1-\lambda i$. By setting $\phi_{2}(a)=\sum_{b, c} \gamma_{a}^{b, c}(b \otimes c)$ for basis elements $a, b, c \in\{1, i\}, d^{2,3}\left(\phi_{2}\right)=0$ implies

$$
\begin{aligned}
\gamma_{1}^{1,1} & =\gamma_{i}^{1, i}=\gamma_{i}^{i, 1}, \\
-\gamma_{i}^{1,1} & =\gamma_{1}^{1, i}=\gamma_{1}^{i, 1},
\end{aligned}
$$

leaving free variables $\gamma_{1}^{1,1}, \gamma_{1}^{i, i}, \gamma_{i}^{1,1}$ and $\gamma_{i}^{i, i}$. The equations $d_{(1)}^{2,2}\left(\phi_{1}, \phi_{2}\right)(1 \otimes 1)=0$ and $d_{(1)}^{2,2}\left(\phi_{1}, \phi_{2}\right)(1 \otimes$ $i)=0$ do not give additional conditions. Assuming that $\phi_{1}(i \otimes i)=\lambda_{i, i}^{1} 1+\lambda_{i, i}^{i} i$, the other two conditions of $d_{(1)}^{2,2}\left(\phi_{1}, \phi_{2}\right)=0$ give

$$
-\lambda+\lambda_{i, i}^{i}+\gamma_{i}^{i, i}-\gamma_{1}^{1, i}=0, \quad \lambda^{\prime}+\lambda_{i, i}^{1}+\gamma_{1}^{i, i}+\gamma_{i}^{1, i}=0
$$

that can be rewritten with Eqns. (5) and (6) as

$$
\begin{aligned}
-\lambda+\lambda_{i, i}^{i}+\gamma_{i}^{i, i}+\gamma_{i}^{1,1} & =0 \\
\lambda^{\prime}+\lambda_{i, i}^{1}+\gamma_{1}^{i, i}+\gamma_{1}^{1,1} & =0
\end{aligned}
$$

Since $\operatorname{dim}\left(Z_{f}^{2}(A ; A)\right)$ is equal to the number of variables $\left(\lambda, \lambda^{\prime}, \lambda_{i, i}^{1}, \lambda_{i, i}^{i}, \gamma_{1}^{1,1}, \gamma_{1}^{i, i}, \gamma_{i}^{1,1}, \gamma_{i}^{i, i}\right)$ minus the number of equations (Eqns. (7) and (죠 ) ), we obtain $\operatorname{dim}\left(Z_{f}^{2}(A ; A)\right)=6$. Hence, together with $B_{f}^{2}(\mathbb{C} ; \mathbb{C}) \cong \mathbb{R}^{2}$, we obtain the stated results.

Example 3.18 For $A=k[x] /\left(x^{2}\right)$ in Example [2.2, we have

$$
H_{f}^{1}(A ; A)=\left\{\begin{array}{lll}
0 & \text { if } & \operatorname{char}(k) \neq 2 \\
k & \text { if } & \operatorname{char}(k)=2
\end{array} \quad, \quad Z_{f}^{2}(A ; A)=k^{6}, \quad H_{f}^{2}(A ; A)=\left\{\begin{array}{ccc}
k^{4} & \text { if } & \operatorname{char}(k) \neq 2 \\
k^{5} & \text { if } & \operatorname{char}(k)=2
\end{array}\right.\right.
$$

Proof. From the proof of Lemma 3.15, the condition $h \in Z_{f}^{1}(A ; A)$ is equivalent to $h(1)=0$, $h(x)=\alpha \cdot 1$ with $2 \alpha=0$, and the following additional conditions that were not used in the proof:

$$
\begin{aligned}
d^{1,1}(h)(x \otimes x) & =h(x) \cdot x+x \cdot h(x)-h(0)=2 x h(x)=0, \\
d^{1,2}(h)(x) & =h(x) \otimes x+x \otimes h(x)-\Delta(h(x))=0,
\end{aligned}
$$


both of which follow from the conditions already stated in the lemma. Hence we obtain $H_{f}^{1}$ as stated. We also have $B^{2}(A ; A) \cong k^{2}$ if $\operatorname{char}(k) \neq 2$ and $B^{2}(A ; A) \cong k$ if $\operatorname{char}(k)=2$.

For 2-cocycles $\phi_{1} \in C^{2,1}(A ; A)$ and $\phi_{2} \in C^{2,2}(A ; A)$, Lemma 3.16 implies that there is $\lambda \in k$ such that

$$
\phi_{1}(1 \otimes x)=\phi_{1}(x \otimes 1)=\lambda x
$$

and $\phi_{1}(1 \otimes 1)=\lambda 1+\lambda^{\prime} x$ for another $\lambda^{\prime} \in k$. Direct calculations show also if $\phi_{2}(a)=\sum_{b, c} \gamma_{a}^{b, c}(b \otimes c)$ then $d^{2,3}\left(\phi_{2}\right)=0$ implies

$$
\gamma_{x}^{1, x}=\gamma_{x}^{x, 1}=0, \quad \gamma_{1}^{1, x}=\gamma_{1}^{x, 1}=\gamma_{x}^{x, x} .
$$

Now let $\phi_{1}(x \otimes x)=\alpha 1+\beta x$. The equation $d_{(1)}^{2,2}\left(\phi_{1}, \phi_{2}\right)(x \otimes 1)=0$ implies $\phi_{1}(x \otimes x)=\gamma_{x}^{1,1} 1-\gamma_{1}^{1,1} x$, (the evaluations at other tensors don't give any extra conditions). In summary we obtain $\phi_{2}(x)=$ $\gamma_{x}^{1,1}(1 \otimes 1)+\gamma_{1}^{1, x}(x \otimes x)$ and $\phi_{2}(1)=\gamma_{1}^{1,1}(1 \otimes 1)+\gamma_{1}^{1, x}(1 \otimes x+x \otimes 1)+\gamma_{1}^{x, x}(x \otimes x)$, in total a six-dimensional solution set parametrized by $\lambda, \lambda^{\prime}, \gamma_{1}^{1,1}, \gamma_{x}^{1,1}, \gamma_{1}^{1, x}$ and $\gamma_{1}^{x, x}$. The result follows.

Example 3.19 For a group algebra $A=k G$ in Example 2.3, we consider the case $G=\mathbb{Z}_{2}$. Then we have

$$
H_{f}^{1}(A ; A)=\left\{\begin{array}{lll}
0 & \text { if } & \operatorname{char}(k) \neq 2 \\
k & \text { if } & \operatorname{char}(k)=2
\end{array}, \quad Z_{f}^{2}(A ; A)=k^{6}, \quad H_{f}^{2}(A ; A)=\left\{\begin{array}{lll}
k^{4} & \text { if } & \operatorname{char}(k) \neq 2 \\
k^{5} & \text { if } & \operatorname{char}(k)=2
\end{array}\right.\right.
$$

Proof. Assuming $d^{1,1}(h)=0$ for $h \in C_{f}^{1}(A ; A)$, Lemma 3.15 implies that $h(1)=0$. The condition $d^{1,1}(h)(x \otimes x)=0$ implies $2 x h(x)=0$, which is equivalent to $2 h(x)=0$. The same condition follows from $d^{1,2}(h)(1)=0$, and the last condition $d^{1,2}(h)(x)=0$ implies that $h(x)=\alpha x$ for some $\alpha \in k$. Thus we obtain $H^{1}$ as stated.

Lemma 3.16 implies that $\phi_{1}$ is given by

$$
\phi_{1}(1 \otimes x)=\phi_{1}(x \otimes 1)=\lambda 1+\lambda^{\prime} x
$$

for some $\lambda, \lambda^{\prime} \in k$, and $\phi_{1}(1 \otimes 1)=\lambda^{\prime} 1+\lambda x$.

From $d^{2,3}\left(\phi_{2}\right)=0$ we obtain

$$
\gamma_{1}^{1,1}=\gamma_{x}^{1, x}=\gamma_{x}^{x, 1}, \quad \gamma_{x}^{1,1}=\gamma_{1}^{1, x}=\gamma_{1}^{x, 1}
$$

Hence, we can write

$$
\begin{aligned}
& \phi_{2}(1)=q(1 \otimes 1)+r(1 \otimes x+x \otimes 1)+\gamma_{1}^{x, x}(x \otimes x), \\
& \phi_{2}(x)=r(1 \otimes 1)+q(1 \otimes x+x \otimes 1)+\gamma_{x}^{x, x}(x \otimes x) .
\end{aligned}
$$

Now let $\phi_{1}(x \otimes x)=\lambda_{x, x}^{1} 1+\lambda_{x, x}^{x} x$. The equation $d^{2,2}\left(\phi_{1}, \phi_{2}\right)=0$ gives by evaluation at the four basis elements the following constraints

$$
\begin{aligned}
& \gamma_{1}^{x, x}+\lambda_{x, x}^{1}=q+\lambda^{\prime}, \\
& \gamma_{x}^{x, x}-\lambda_{x, x}^{x}=r-\lambda .
\end{aligned}
$$

Since $\operatorname{dim}\left(Z_{f}^{2}(A ; A)\right)$ is equal to the number of variables $\left(q, r, \lambda, \lambda^{\prime}, \gamma_{1}^{x, x}, \gamma_{x}^{x, x}, \lambda_{x, x}^{1}, \lambda_{x, x}^{x}\right)$ minus the number of equations (the above two), we obtain $\operatorname{dim}\left(Z_{f}^{2}(A ; A)\right)=6$. Thus we obtain the result.

Remark 3.20 It is interesting that these Frobenius algebras all have the same 1 and 2-dimensional cohomology when $\operatorname{char}(k) \neq 2$. Nevertheless, the free variables are quite a bit different in each computation. We expect that higher-dimensional algebras are cohomologically distinct. 


\section{Yang-Baxter solutions in Frobenius algebras and their cocycle deformations}

In this section, we construct YBE solutions ( $R$-matrices) from skein theoretic methods using maps in Frobenius algebras. We start with the following examples, the first of which is due to [18, and diagrammatic proofs are depicted in Figs. 11, 12, and 13, respectively.

The Yang-Baxter equation (YBE) is formulated as $(R \otimes \mid)(\mid \otimes R)(R \otimes \mid)=(\mid \otimes R)(R \otimes \mid)(\mid \otimes R)$ for $R \in \operatorname{Hom}(V \otimes V, V \otimes V)$, see, for example, [10]. It is often required that $R$ be invertible, but we do not always require the invertibility unless explicitly mentioned. In particular, the solutions in Lemma 4.1 are not invertible. Invertible solutions derived from these are discussed in Proposition 4.3 .

Lemma 4.1 For any Frobenius algebra $X, R=\Delta \mu$ is a solution to the Yang-Baxter equation. For any symmetric Frobenius algebra $X, R_{1}=\tau \Delta \mu$ and $R_{2}=(\mu \otimes \mid)(\mid \otimes \tau)(\Delta \otimes \mid)$ are solutions to the $Y B E$ as well.

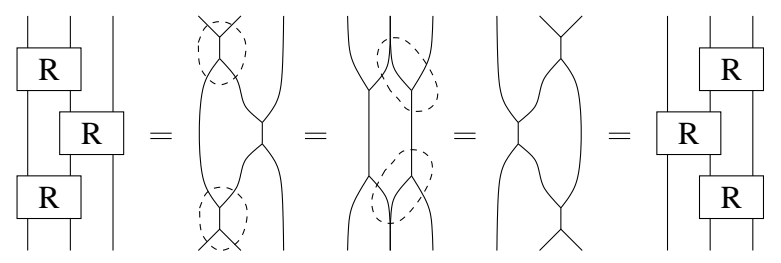

Figure 11: A solution to YBE in Frobenius algebras

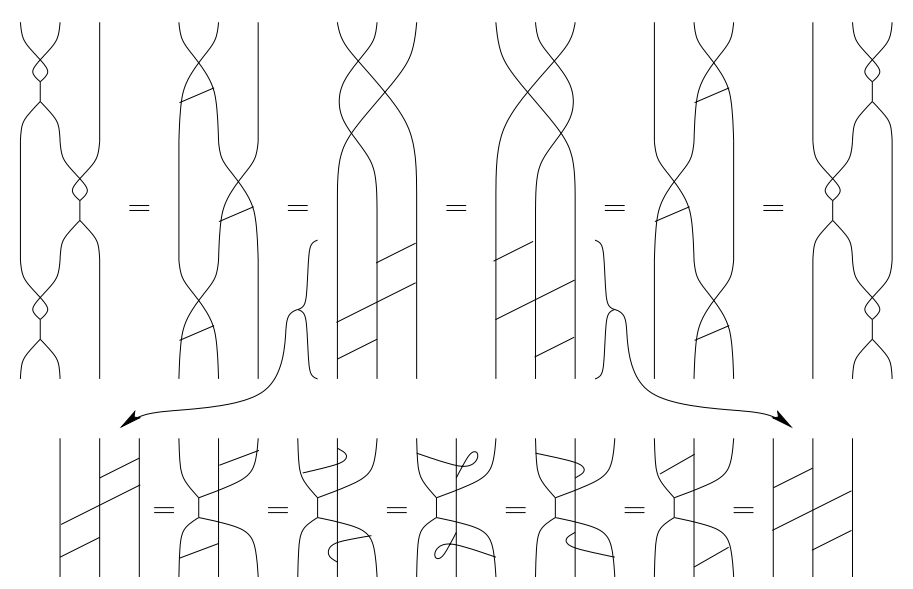

Figure 12: A solution to YBE in symmetric Frobenius algebras

Example 4.2 For a group ring $X=k G$ in Example 2.3, the $R$-matrix of type $\Delta \mu$ is computed by $R(x \otimes y)=(\Delta \mu)(x \otimes y)=\sum_{z w=x y} z \otimes w$ for $x, y \in G$. 


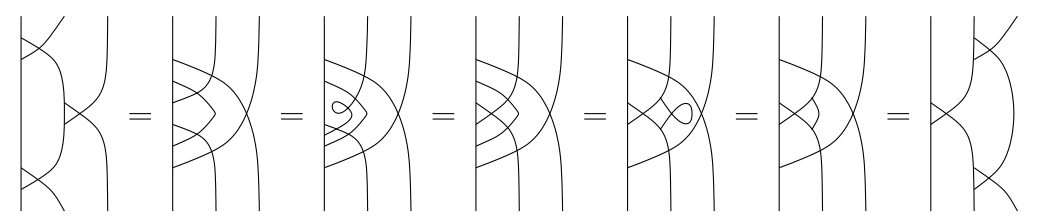

Figure 13: Another solution to YBE in symmetric Frobenius algebras

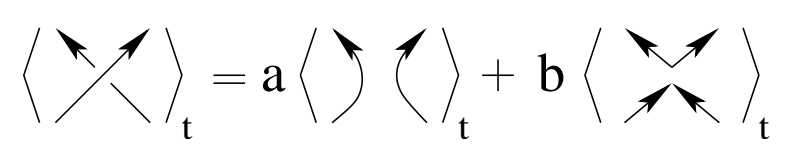

Figure 14: A skein relation

We define two $R$-matrices $R$ and $R^{\prime}$ by skein relations as depicted in Fig. 14, which are written as follows.

$$
\begin{aligned}
R & =\left.A\right|^{\otimes 2}+B(\gamma \beta)+C(\Delta \mu)+T(\tau), \\
R^{\prime} & =\left.A^{\prime}\right|^{\otimes 2}+B^{\prime}(\gamma \beta)+C^{\prime}(\Delta \mu)+T^{\prime}(\tau) .
\end{aligned}
$$

We call this the Frobenius skein relation, and below we derive conditions for it to satisfy the YBE. We also suppose that the inverse of $R$ is given by $R^{\prime}$, and compute the conditions for this requirement. Note that instead of the map $\Delta \mu$, the two other solutions in Lemma 4.1 can be used to define similar skein relations, and these possibilities might deserve further study.

Note that $\beta \gamma(1)$ is an element of $k$ which we denote by $\delta_{0}$. In the following, sometimes we make the assumption that $\mu \Delta=\delta_{1} \mid$ for some $\delta_{1} \in k$ for computational simplicity. (Recall that in general it is true that $\mu \Delta=\delta_{h} \mid$ holds but $\delta_{h} \in A$ is a central element.) This holds for some of the examples of Frobenius algebras, such as group algebras. Under this assumption one obtains the following relation:

$$
\begin{gathered}
\delta_{0}=\beta \gamma=\epsilon \mu \Delta \eta(1)=\epsilon\left(\delta_{1} 1\right)=\delta_{1} \epsilon(1) \\
Y=Y=Q=\lambda=\lambda)
\end{gathered}
$$

Figure 15: A few formulas

In Fig. 15] a few direct calculations are depicted that will be used below. Using this notation, one calculates the condition for $R^{\prime}$ to be the inverse of $R$. A calculation is illustrated in Fig. 16 for the condition that $R^{\prime}=R^{-1}$.

In Figs. 17 and 18 the YBE is formulated diagrammatically for the above defined skein relation. 


$$
\begin{aligned}
& \left\langle=(\mathrm{A}||+\mathrm{B} \underset{\sim}{\cup}+\mathrm{C} X+\mathrm{T} X)\left(\mathrm{A}^{\prime}||+\mathrm{B}^{\prime} \underset{\sim}{\cup}+\mathrm{C}^{\prime} X+\mathrm{T}^{\prime} X\right)\right. \\
& =\left(\mathrm{AA}^{\prime}+\mathrm{TT}^{\prime}\right)||+\left(\mathrm{AB}^{\prime}+\mathrm{A}^{\prime} \mathrm{B}+\delta_{0} \mathrm{BB}^{\prime}\right) \stackrel{\cup}{\sim} \\
& +\mathrm{BT}^{\prime} \underset{\gamma}{\cup}+\mathrm{B}^{\prime} \mathrm{T} \underset{\cap}{\gamma}+\mathrm{B}^{\prime} \underset{\mathrm{q}}{\cup}+\mathrm{B}^{\prime} \mathrm{C} \stackrel{\mathrm{d}}{\sim} \\
& +\left(\mathrm{AC}^{\prime}+\mathrm{A}^{\prime} \mathrm{C}\right) X+\mathrm{CT}^{\prime} Y+\mathrm{C}^{\prime} \mathrm{T} Y+\mathrm{CC}^{\prime} \stackrel{Y}{Y}+\left(\mathrm{AT}^{\prime}+\mathrm{A}^{\prime} \mathrm{T}\right) X
\end{aligned}
$$

Figure 16: The skein relation of the inverse

Proposition 4.3 Suppose the Frobenius algebra $X$ over a field $k$ satisfies $\mu \Delta=\delta_{1} \mid$ for some $\delta_{1} \in k$. Then the $R$-matrix defined by the Frobenius skein relation (11), with the inverse $R^{-1}=R^{\prime}$ defined by the relation (12), gives a solution to the $Y B E$ if the following hold:

(i) $C=T=0, C^{\prime}=T^{\prime}=0, A^{2}+B^{2}+\delta_{0} A B=0, A^{\prime 2}+B^{\prime 2}+\delta_{0} A^{\prime} B^{\prime}=0$, and $A B^{\prime}+A^{\prime} B+\delta_{0} B B^{\prime}=0$.

(ii) $X$ is commutative, $A=B=0, A^{\prime}=B^{\prime}=0, T T^{\prime}=1$ and $C T^{\prime}+C^{\prime} T+\delta_{1} C C^{\prime}=0$.

Proof. We obtain the conditions $A^{2} T=0$ and $B^{2} T=0$ by comparing the coefficients of the following terms for both sides of the equation respectively (see Figs. 17] and 18):

$$
\begin{aligned}
& |\otimes \tau, \tau \otimes|, \\
& (\mid \otimes \tau)(\gamma \otimes \mid)(\beta \otimes \mid),(\tau \otimes \mid)(\mid \otimes \gamma)(\mid \otimes \beta),(\beta \otimes \mid)(\gamma \otimes \mid)(\mid \otimes \tau), \quad(\mid \otimes \beta)(\mid \otimes \gamma)(\mid \otimes \tau) .
\end{aligned}
$$

Assuming that variables take values in the field $k$, we obtain either $T=0$ or $A=B=0$.

Assume $T=0$, and also assume that $R^{-1}$ satisfies the similar condition, $T^{\prime}=0$. For $R$ we obtain

$$
\begin{aligned}
B\left(A^{2}+B^{2}+\delta_{0} A B+\delta_{1} B C+2 \delta_{1} A C\right) & =0, \\
A C\left(A+\delta_{1} C\right) & =0 \\
B C\left(B+\delta_{1} C\right) & =0
\end{aligned}
$$

that are derived from comparing the coefficients of the following maps:

$$
\begin{aligned}
& |\otimes \gamma \beta, \gamma \beta \otimes|, \\
& (\mid \otimes \Delta \mu),(\Delta \mu \otimes \mid), \\
& (\mid \otimes \gamma) \mu(\mu \otimes \mid),(\gamma \otimes \mid) \mu(\mu \otimes \mid),(\Delta \otimes \mid) \Delta(\mid \otimes \beta),(\mid \otimes \Delta) \Delta(\beta \otimes \mid) .
\end{aligned}
$$

It is, then, easy to see from these equations and from Figs. 17and 18 that the conditions $C=T=0$ and $A^{2}+B^{2}+\delta_{0} A B=0$ give solution $R$ to the YBE. Similarly from the condition depicted in Fig. 16, it follows that $R^{\prime}$ gives the inverse of $R$ with the conditions $C^{\prime}=T^{\prime}=0, A^{\prime 2}+B^{\prime 2}+\delta_{0} A^{\prime} B^{\prime}=0$ and $A B^{\prime}+A^{\prime} B+\delta_{0} B B^{\prime}=0$.

In this paragraph we observe that the condition $C=0$, in fact, follows from the required conditions assuming that variables take values in a field $k$. Suppose $C \neq 0$. Then Eqns. (15) and (16) require $A=B=-\delta_{1} C$ and $A^{\prime}=B^{\prime}=-\delta_{1} C^{\prime}$. Then Eqn. (14) implies $\delta_{0}=1$, and from Eqn. (13) $\left(\delta_{0}=\delta_{1} \epsilon(1)\right)$, we have $\delta_{1}=\epsilon(1)^{-1}$. The inverse formula (Fig. 16) in this case $(C=T=0)$ 


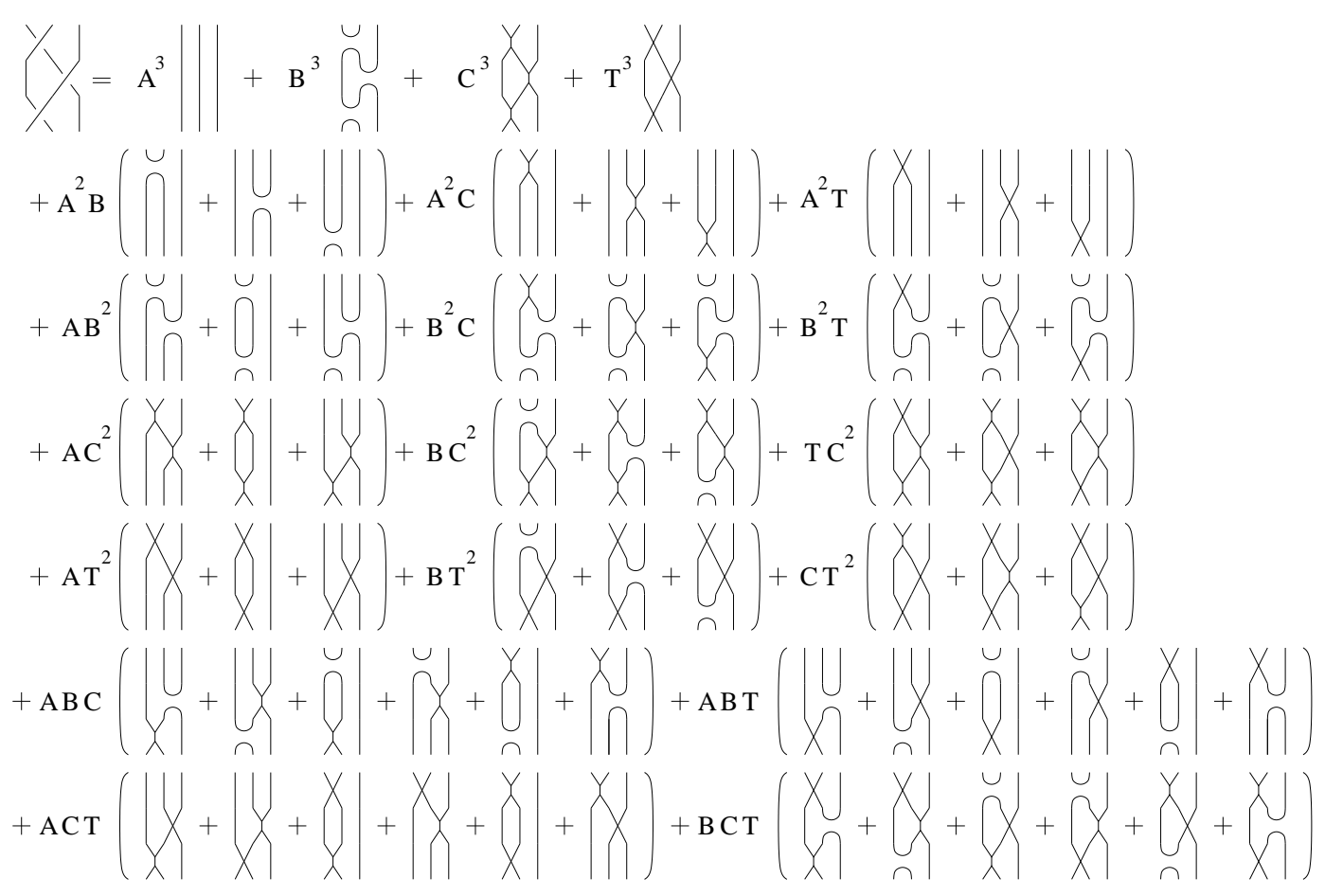

Figure 17: YBE for a skein, LHS

requires $A B^{\prime}+A^{\prime} B+\delta_{0} B B^{\prime}=0$, which reduces to $\delta_{1} C C^{\prime}=0$, a contradiction. Hence we have $C=0$.

Next we consider the case $A=B=0$, and assume that $R^{-1}$ is in the same form, $A^{\prime}=B^{\prime}=0$. Then the commutativity implies LHS=RHS in Figs. 17 and 18, using formulas depicted in Fig. 15, The inverse condition in Fig. 16 and the definition $R^{-1}=C^{\prime}(\Delta \mu)+T^{\prime} \tau$ imply $T T^{\prime}=1$ and $C T^{\prime}+C^{\prime} T+\delta_{1} C C^{\prime}=0$. This leads to Case (ii).

Using 2-cocycles, we construct new $R$-matrices from old by deformation as follows. Let $X$ be a Frobenius algebra over $k$. Suppose $R$ is defined by the Frobenius skein relation that satisfies the conditions in Proposition 4.3, so that $R$ is a solution to the YBE on $X$.

Let $\hat{X}=A \otimes k[[t]] /\left(t^{2}\right)$. Then $\hat{X}$ is regarded as $\left(k[t] /\left(t^{2}\right)\right)$-module. Extend the maps $\mu$ and $\Delta$ to $\hat{X}$. From the deformation interpretation of 2-cocycles in Section 3.1, we have the following.

Theorem 4.4 Let $X$ be a commutative, and therefore cocommutative, Frobenius algebra. Suppose $\phi_{i} \in C^{2}(X ; X), i=1,2$, are Frobenius 2 -cochains satisfying all the 2 -cocycle conditions $d^{2,1}=$ $d_{(1)}^{2,2}=d_{(2)}^{2,2}=d^{2,3}=0$. Define $R_{\phi_{1}, \phi_{2}}: \hat{X} \otimes \hat{X} \rightarrow \hat{X} \otimes \hat{X}$ by

$$
R_{\phi_{1}, \phi_{2}}=C\left(\left(\Delta+t \phi_{2}\right)\left(\mu+t \phi_{1}\right)\right)+T(\tau) .
$$

Then $R_{\phi_{1}, \phi_{2}}$ is a solution to the YBE if the following conditions are satisfied: $\left(\mu+t \phi_{1}\right)\left(\Delta+t \phi_{2}\right)=\delta_{1} \mid$ on $\hat{X}$ for some $\delta_{1} \in k[t] /\left(t^{2}\right), \phi_{1} \tau=\phi_{1}$, and $\tau \phi_{2}=\phi_{2}$.

Proof. This is a repetition of the proof of Proposition 4.3 Case (ii), using the deformation interpretations of 2-cocycle conditions. The associativity, coassociativity, and Frobenius compatibility 


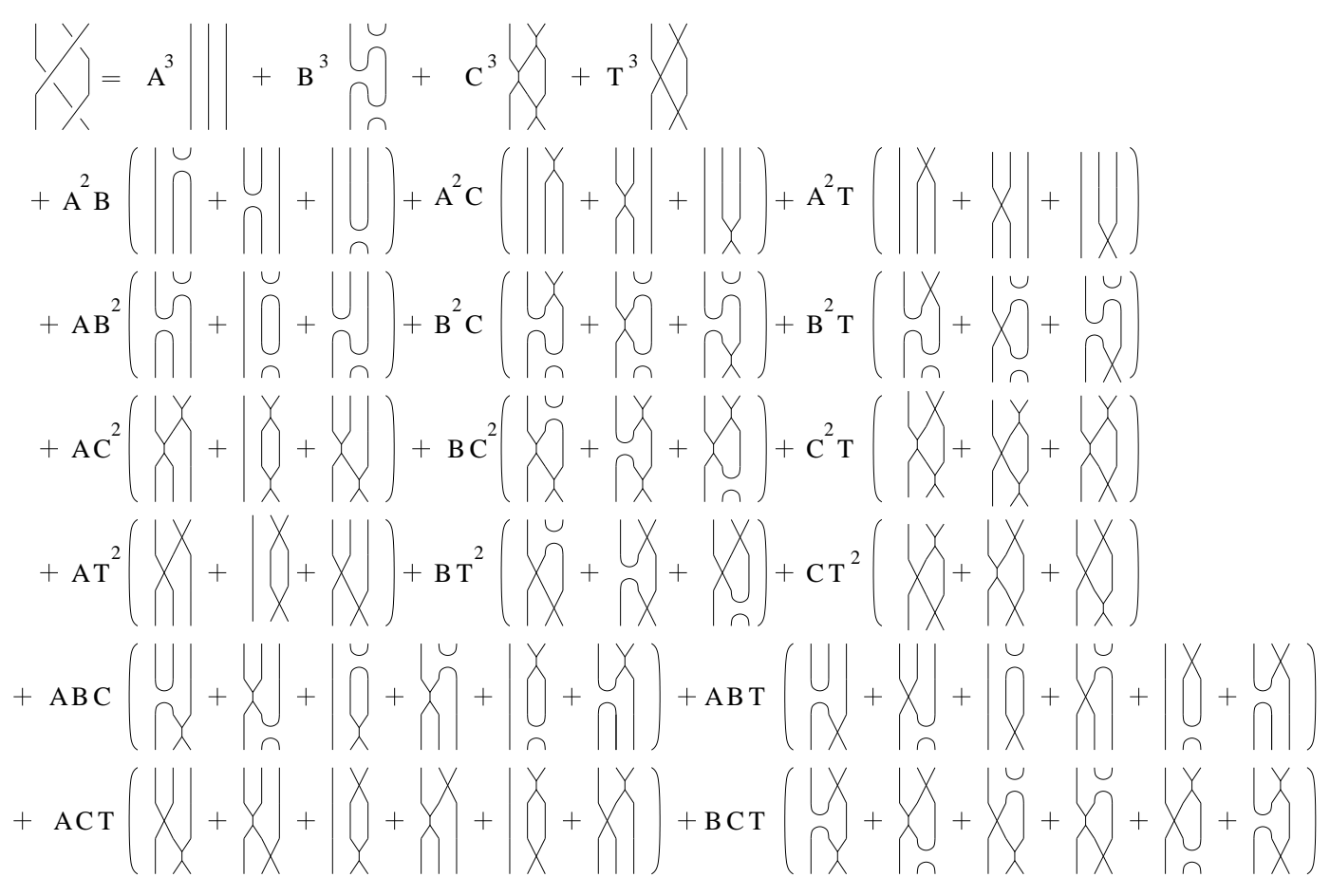

Figure 18: YBE for a skein, RHS

conditions for $\mu+t \phi_{1}$ and $\Delta+t \phi_{2}$ follow from the 2-cocycle conditions in Lemma 3.2. The conditions $\phi_{1} \tau=\phi_{1}$ and $\tau \phi_{2}=\phi_{2}$ correspond to the commutativity.

Example 4.5 For $X=\mathbb{C}$ in Example 2.1, the general solutions for the 2-cocycles $\phi_{1}$ and $\phi_{2}$ with $d^{2,1}=d_{(1)}^{2,2}=d_{(2)}^{2,2}=d^{2,3}=0$ found in Example 3.17 satisfy the condition (ii) in Theorem 4.4 $\phi_{1} \tau=\phi_{1}, \tau \phi_{2}=\phi_{2}$. We check the condition $\left(\mu+t \phi_{1}\right)\left(\Delta+t \phi_{2}\right)=\delta_{1} \mid$ on $\hat{X}$ for some $\delta_{1} \in k[t] /\left(t^{2}\right)$. One computes:

$$
\begin{aligned}
\left(\mu+t \phi_{1}\right)\left(\Delta+t \phi_{2}\right)(1) & =2+t\left[\left(\gamma_{1}^{1,1}-\gamma_{1}^{i, i}+\lambda^{\prime}-\lambda_{i, i}^{1}\right)+\left(\gamma_{1}^{1, i}+\gamma_{1}^{i, 1}-\lambda-\lambda_{i, i}^{i}\right) i\right], \\
\left(\mu+t \phi_{1}\right)\left(\Delta+t \phi_{2}\right)(i) & =2 i+t\left[\left(\gamma_{i}^{1,1}-\gamma_{i}^{i, i}+2 \lambda\right)+\left(\gamma_{i}^{1, i}+\gamma_{i}^{i, 1}+2 \lambda^{\prime}\right) i\right] .
\end{aligned}
$$

Thus the general 2-cocycles satisfy $\left(\mu+t \phi_{1}\right)\left(\Delta+t \phi_{2}\right)=\delta_{1} \mid$ if and only if the above two values are multiples of 1 and $i$, respectively, by the same element $\delta_{1} \in k[t] /\left(t^{2}\right)$. This condition is written as

$$
\begin{aligned}
\gamma_{1}^{1, i}+\gamma_{1}^{i, 1}-\lambda-\lambda_{i, i}^{i} & =0 \\
\gamma_{i}^{1,1}-\gamma_{i}^{i, i}+2 \lambda & =0, \\
\gamma_{1}^{1,1}-\gamma_{1}^{i, i}+\lambda^{\prime}-\lambda_{i, i}^{1} & =\gamma_{i}^{1, i}+\gamma_{i}^{i, 1}+2 \lambda^{\prime} .
\end{aligned}
$$

For Eqn. (19), Eqns. (5) and (8) imply

$$
\gamma_{1}^{1,1}-\gamma_{1}^{i, i}+\lambda^{\prime}-\lambda_{i, i}^{1}=-2\left(\gamma_{1}^{i, i}+\lambda_{i, i}^{1}\right)=\gamma_{i}^{1, i}+\gamma_{i}^{i, 1}+2 \lambda^{\prime}
$$

so that Eqn. (19) is redundant and we obtain $\delta_{1}=2\left[1-t\left(\lambda_{i, i}^{1}+\gamma_{1}^{i, i}\right)\right]$. 
Thus, from the computation in Example 3.17, the general 2-cocycle satisfying the conditions in Theorem 4.4 Case (ii) has variables $\left(\lambda, \lambda^{\prime}, \lambda_{i, i}^{1}, \lambda_{i, i}^{i}, \gamma_{1}^{1,1}, \gamma_{1}^{i, i}, \gamma_{i}^{1,1}, \gamma_{i}^{i, i}\right)$, with Eqns. (17), (8), (17), and (18). Equations (17) and (18) reduce with Eqn. (7) to the same equation

$$
3 \gamma_{i}^{1,1}+2 \lambda_{i, i}^{i}+\gamma_{i}^{i, i}=0
$$

so the deformed $R$ matrix in this case has 5 free variables.

Example 4.6 For $X=k \mathbb{Z}_{2}$ in Example 2.3, the general solutions for the 2-cocycles $\phi_{1}$ and $\phi_{2}$ with $d^{2,1}=d_{(1)}^{2,2}=d_{(2)}^{2,2}=d^{2,3}=0$ found in Example 3.19 satisfy the condition (ii) in Theorem 4.4; $\phi_{1} \tau=\phi_{1}, \tau \phi_{2}=\phi_{2}$. We check the condition $\left(\mu+t \phi_{1}\right)\left(\Delta+t \phi_{2}\right)=\delta_{1} \mid$ on $\hat{X}$ for some $\delta_{1} \in k[t] /\left(t^{2}\right)$. One computes:

$$
\begin{aligned}
& \left(\mu+t \phi_{1}\right)\left(\Delta+t \phi_{2}\right)(1)=2+t\left[\left(q+\gamma_{1}^{x, x}+\lambda^{\prime}+\lambda_{x, x}^{1}\right)+\left(2 r+\lambda+\lambda_{x, x}^{x}\right) x\right], \\
& \left(\mu+t \phi_{1}\right)\left(\Delta+t \phi_{2}\right)(x)=2 x+t\left[\left(r+\gamma_{x}^{x, x}+2 \lambda\right)+\left(2 q+2 \lambda^{\prime}\right) x\right] .
\end{aligned}
$$

Thus the general 2-cocycles satisfy $\left(\mu+t \phi_{1}\right)\left(\Delta+t \phi_{2}\right)=\delta_{1} \mid$, if and only if the above two values are multiples of 1 and $x$, respectively by the same element $\delta_{1} \in k[t] /\left(t^{2}\right)$. This condition is written as

$$
\begin{aligned}
2 r+\lambda+\lambda_{x, x}^{x} & =0, \\
r+\gamma_{x}^{x, x}+2 \lambda & =0, \\
\gamma_{1}^{x, x}+\lambda^{\prime}+\lambda_{x, x}^{1} & =q+2 \lambda^{\prime} .
\end{aligned}
$$

Using Eqns. (9) and (10), one obtains $\lambda_{x, x}^{x}=3 \lambda+2 \gamma_{x}^{x, x}$ and one can compute $\delta_{1}=2\left[1+t\left(\lambda_{x, x}^{1}+\gamma_{1}^{x, x}\right)\right]$. Since the equations listed for Example 3.19 are the only equations we need for this example as well, the deformed $R$ matrix has 5 free variables.

\section{Conclusion}

This paper contains a study of the cohomology of Frobenius algebras initiated from the point of view of deformation theory as explicated by diagrammatic techniques. One reason for developing this theory diagrammatically is that we obtain cocycle deformations of $R$-matrices, and anticipate topological applications.

Several problems remain. Clearly computations of cohomology for Frobenius algebras formed as matrix algebras would be especially interesting if these could be done in a fashion that encompassed all dimensions. The relationship between this Frobenius cohomology theory and the cohomology of the adjoint map in a Hopf algebra deserves study. Finally, an understanding of the possible knot invariants defined by YBE solutions defined by skein relations among maps in Frobenius algebras would be very interesting.

\section{References}

[1] Asaeda, M.; Frohman, C., A note on the Bar-Natan skein module, Preprint, available at: arXiv:math/0602262. 
[2] Carter, J.S.; Jelsovsky, D.; Kamada, S.; Langford, L.; Saito, M., Quandle cohomology and state-sum invariants of knotted curves and surfaces, Trans. Amer. Math. Soc. 355 (2003), no. 10, 3947-3989.

[3] Carter, J.S.; Crans, A.; Elhamdadi, M.; Saito, S., Cohomology of Categorical SelfDistributivity, Preprint, available at arXiv:math.GT/0607417.

[4] Carter, J.S.; Crans, A.; Elhamdadi, M.; Saito, S., Cohomology of the adjoint of Hopf algebras, Preprint, available at arXiv:0705.3231,

[5] Carter, J.S.; Elhamdadi, M.; Nikiforou, M.A.; Saito, S., Extensions of quandles and cocycle knot invariants, Journal of Knot Theory and Its Ramifications, Vol. 12, No. 6 (2003) 725-738.

[6] Carter, J.S.; Elhamdadi, M.; Saito, S., Twisted Quandle homology theory and cocycle knot invariants, Algebraic and Geometric Topology (2002) 95-135.

[7] Carter, J.S.; Flath, D.E.; Saito, S., Classical and Quantum 6j Symbols, Mathematical notes, vol. 43, Princeton University Press, 1995.

[8] Gerstenharber, M; Schack, S.D., Bialgebra cohomology, deformations, and quantum groups, Proc. Nat. Acad. Sci. U.S.A., 87 (1990), 478-481.

[9] Joyce, D., A classifying invariant of knots, the knot quandle, J. Pure Appl. Alg., 23, 37-65.

[10] Kauffman, L.H., Knots and Physics, World Scientific, Series on knots and everything, vol. 1, 1991.

[11] Kauffman, L.H.; Radford, D.E., Invariants of 3-manifolds derived from finite-dimensional Hopf algebras, J. Knot Theory Ramifications 4 (1995), 131-162.

[12] Khovanov, M., A categorification of the Jones polynomial, Duke Math. J. 101(3) (1999), $359-426$.

[13] Kock, J., Frobenius algebras and 2D topological quantum field theories, London Mathematical Society Student Texts (No. 59), Cambridge University Press, 2003.

[14] Kuperberg, G., Involutory Hopf algebras and 3-manifold invariants, Internat. J. Math. 2 (1991), 41-66.

[15] Kuperberg, G., Noninvolutory Hopf algebras and 3-manifold invariants, Duke Math. J. 84 (1996), 83-129.

[16] Markl, M.; Stasheff, J.D., Deformation theory via deviations, J. Algebra 170 (1994), 122-155.

[17] Ohtsuki, T., Invariants of 3-manifolds derived from universal invariants of framed links, Math. Proc. Cambridge Philos. Soc. 117 (1995), 259-273.

[18] Stolin, A., Frobenius algebras and the Yang-Baxter equation, New symmetries in the theories of fundamental interactions (Karpacz, 1996), 93-97, PWN, Warsaw, 1997. 